\title{
Assessment of the implementation level of the guidelines for secondary prevention of cardiovascular disease in everyday clinical practice
}

\author{
Agata Krawczyk-Ożóg ${ }^{1,2}$, Anna Płotek¹, Mateusz Hołda2,3,4, Dariusz Dudek¹, Renata Rajtar-Salwa \\ 1 Department of Cardiology and Cardiovascular Interventions, University Hospital, Kraków, Poland \\ 2 HEART - Heart Embryology and Anatomy Research Team, Department of Anatomy, Jagiellonian University Medical College, Kraków, Poland \\ 3 Division of Cardiovascular Sciences, The University of Manchester, Manchester, United Kingdom \\ 4 Department of Diagnostics, John Paul II Hospital in Kraków, Kraków, Poland
}

\section{KEY WORDS}

cardiovascular diseases, physical activity, secondary prevention, tobacco smoking
Correspondence to: Agata Krawczyk-Ożóg, MD, PhD Department of Cardiology and Cardiovascular Interventions, ul. Jakubowskiego 2 , 30-688 Kraków, Poland, phone: +48124002267, email: krawczyk.ozog@gmail.com Received: September 9, 2020. Revision accepted: February 13, 2021.

Published online: March 3, 2021. Kardiol Pol. 2021; 79 (4): 434-441 doi:10.33963/KP.15856

Copyright by the Author(s), 2021

\section{ABSTRACT}

BACKGROUND The goal of secondary prevention is to hinder the recurrence of cardiovascular events in patients already diagnosed with cardiovascular diseases.

AIMS We aimed to assess the level of adherence to guidelines for secondary prevention of cardiovascular disease in everyday clinical practice.

METHODS This was a single-center retrospective analysis of 460 consecutive rehospitalized patients previously diagnosed with coronary artery disease. The presence of main risk factors for cardiovascular disease was analyzed in this cohort.

RESULTS Overall, $80.7 \%$ of patients did not comply with the body mass index recommendations. Among nondiabetic patients, $43.5 \%$ exceeded the recommended blood glucose level and $55.5 \%$ of diabetic patients exceeded the recommended level of glycated hemoglobin. Total cholesterol level was higher than recommended in $13.5 \%$ of patients, the level of low-density lipoprotein (LDL) cholesterol was exceeded in $78.7 \%$ individuals, and the level of triglycerides was over the limit in $30.2 \%$ of patients. Systolic and / or diastolic blood pressure higher than or equal to $140 / 90 \mathrm{~mm} \mathrm{Hg}$ was recorded in $41.3 \%$ of patients. Low level of physical activity was declared by $56.7 \%$ of the studied patients and $14.6 \%$ of them admitted to being current tobacco smokers. No patient fulfilled all of the main prevention goals (body weight, no smoking, LDL cholesterol level, glucose level, systolic and / or diastolic blood pressure) and in $10.2 \%$ of cases none of the above-mentioned criteria were achieved. Significant difference in the implementation level of the guidelines was found between the sexes, with men showing lower adherence than women. CONCLUSIONS The level of adherence to the guidelines for secondary prevention of coronary artery disease was extremely low, with men being worse responders than women.

INTRODUCTION Cardiovascular diseases have been the leading cause of morbidity, disability, and mortality worldwide, despite the observed development of new treatment methods as well as improvement in outcomes. ${ }^{1,2}$ Secondary prevention aims to prevent the recurrence of cardiovascular events in patients already diagnosed with cardiovascular diseases. Individuals with established cardiovascular disease are at a high risk for the occurrence of undesirable events such as myocardial infarction, stroke, and death; thus, they constitute the highest clinical priority for secondary prevention. ${ }^{3}$ The main foundations of secondary prevention of cardiovascular diseases include optimal pharmacotherapy and nonpharmacological modification of the risk factors, such as lifestyle change, increase in physical activity, optimal diet, and quitting unhealthy habits like smoking. ${ }^{4} \mathrm{Nev}-$ ertheless, establishment and optimal control of the risk factors in this cohort is still challenging. ${ }^{5}$ 


\section{WHAT'S NEW?}

Identification of the fields of deviation from the official clinical practice guidelines for secondary prevention of cardiovascular disease may provide valuable conclusions on how to improve the quality of secondary prevention of cardiovascular events. Our study demonstrated that the level of adherence to the guidelines in everyday clinical practice was extremely low. No patient fulfilled all of the prevention goals. Therefore, more attention should be paid to proper implementation of the guidelines and patients' education to prevent progression of cardiovascular disease.

Adequate control of hypertension, dyslipidemia, diabetes, and heart failure as well as significant lifestyle modifications allow to reduce the risk of recurrent cardio- and cerebrovascular ischemic events. Nevertheless, many studies have shown that a large majority of patients with coronary diseases did not achieve the guideline standards for secondary prevention. ${ }^{6-8}$ Individual patient's compliance played the most important role in the implementation of preventive measures. However, large discrepancies in the realization of secondary prevention practices might be observed within different health systems and even between individual clinicians, which could lead to negative consequences for the patients. ${ }^{9,10}$ Identification of the fields of deviation from the official clinical practice guidelines might provide valuable conclusions on how to improve the quality of secondary prevention of cardiovascular events. Therefore, we aimed to assess the level of adherence to the guidelines for secondary prevention of cardiovascular disease in everyday clinical practice and compare some cardiovascular risk factors between the sexes.

METHODS Study population The present study was a single-center retrospective analysis of 460 consecutive patients (women, 26.1\%) with previously diagnosed coronary artery disease who were rehospitalized due to subsequent cardiovascular event from June 2016 to December 2018 at the 2nd Department of Cardiology and Cardiovascular Interventions, University Hospital in Kraków, Poland-a tertiary referral medical center. All the patients had a history of previous percutaneous coronary intervention and / or coronary artery bypass grafting or coronary artery disease treated pharmacologically. The median time from last to current hospitalization was 10 months (range, 1-364 months) and median time from the diagnosis of coronary artery disease to current hospitalization was 58 months (range, 1-461 months). This information was not available for 59 patients.

Data collection and interpretation Demographics, baseline characteristics, and medical history data were collected from all patients. History of any cardiac interventions (surgical or percutaneous) or presence of any implantable cardiac devices was noted. The presence of other comorbidities, especially chronic heart failure with reported symptoms assessed with the New York Heart Association Functional Classification, ischemic heart disease with symptoms evaluated with the Canadian Cardiovascular Society Angina Grading Scale, atrial fibrillation, diabetes mellitus, pulmonary disorders, peptic ulcer disease, thyroid disorders, previous cerebrovascular accidents (transient ischemic attack and/or stroke), and chronic kidney disease was recorded based on patients' medical history. Family history of early cardiovascular disease (in men younger than 55 and women younger than 65 years) was also investigated.

The height and weight of patients were measured by medical staff on admission. The body mass index (BMI) was calculated as the body mass in kilograms divided by the square of the height in meters and expressed in units of $\mathrm{kg} / \mathrm{m}^{2}$. Patients were classified as underweight (BMI $\left.\leq 18.4 \mathrm{~kg} / \mathrm{m}^{2}\right)$, normal weight (BMI 18.5-24.9 $\mathrm{kg} / \mathrm{m}^{2}$ ), overweight (BMI $25-29.9 \mathrm{~kg} / \mathrm{m}^{2}$ ), and obese (BMI $\geq 30 \mathrm{~kg} / \mathrm{m}^{2}$ ), with the latter group further divided into class I (BMI $30-34.9 \mathrm{~kg} / \mathrm{m}^{2}$ ), class II (BMI $35-39.9 \mathrm{~kg} / \mathrm{m}^{2}$ ), and class III (BMI $\geq 40 \mathrm{~kg} / \mathrm{m}^{2}$ ), according to the guidelines of the World Health Organization and National Institutes of Health. ${ }^{11}$ Additionally, waist circumference was measured. Abdominal overweight was defined as waist circumference of 95 to $102 \mathrm{~cm}$ in men and 81 to $88 \mathrm{~cm}$ in women. Abdominal obesity was considered present if waist circumference was greater than $102 \mathrm{~cm}$ in men and greater than $88 \mathrm{~cm}$ in women. ${ }^{12}$

Fasting blood samples were collected on patient admission as well. The levels of low-density lipoprotein (LDL) cholesterol (target value, $<1.4 \mathrm{mmol} / \mathrm{l}$ ), ${ }^{13}$ total cholesterol (reference range, 3.2-5.2 mmol/l), high-density lipoprotein (HDL) cholesterol (target value, $>1 \mathrm{mmol} / \mathrm{l}$ in men and $>1.2 \mathrm{mmol} / \mathrm{l}$ in women), triglycerides (target value, $\leq 1.7 \mathrm{mmol} / \mathrm{l}),{ }^{14}$ fasting glucose (in patients without diabetes mellitus; reference range, $3.9-5.5 \mathrm{mmol} / \mathrm{l}$ ), and glycated hemoglobin $\left(\mathrm{HbA}_{1 \mathrm{c}}\right)$ (in diabetic patients; target value, $\left.\leq 7 \%\right)^{15}$ were measured. Blood pressure measurements were taken on the second day of hospitalization by medical staff. Elevated blood pressure was defined as systolic blood pressure greater than or equal to $140 \mathrm{~mm} \mathrm{Hg}$ and / or diastolic blood pressure greater than or equal to $90 \mathrm{~mm} \mathrm{Hg} .{ }^{16}$ Left ventricular ejection fraction was assessed using transthoracic echocardiography.

Dietary habits were assessed using a specially-designed, study-dedicated questionnaire. It was focused on the consumption of saturated fatty acids, fiber, fruit, vegetables, fish, salt, and alcohol (Supplementary material). The level of physical activity was evaluated by the short form of the International Physical Activity Questionnaire, ${ }^{17,18}$ which delineates 3 levels of physical activity: low, 
TABLE 1 Baseline clinical and demographic characteristics of patients $(n=460)$

\section{Variable}

Value

\begin{tabular}{|c|c|c|}
\hline \multicolumn{2}{|c|}{ Age, y, mean (SD) } & $66.4(9.9)$ \\
\hline \multicolumn{2}{|c|}{ Male sex } & $340(73.9)$ \\
\hline \multicolumn{2}{|c|}{ Previous myocardial infarction } & $287(62.4)$ \\
\hline \multicolumn{2}{|c|}{ Previous percutaneous coronary intervention } & $373(81.1)$ \\
\hline \multicolumn{2}{|c|}{ Previous coronary artery bypass grafting } & $49(10.7)$ \\
\hline \multirow[t]{5}{*}{ CCS } & None & $50(10.9)$ \\
\hline & I & $133(28.9)$ \\
\hline & II & $157(34.1)$ \\
\hline & III & $92(20)$ \\
\hline & IV & $28(6.1)$ \\
\hline \multirow[t]{5}{*}{ NYHA } & none & $103(22.4)$ \\
\hline & I & $133(28.9)$ \\
\hline & II & $151(32.8)$ \\
\hline & III & $59(12.8)$ \\
\hline & IV & $14(3)$ \\
\hline \multicolumn{2}{|c|}{ Left ventricular ejection fraction, $\%$, median (IQR) } & $55(43-60)$ \\
\hline \multicolumn{2}{|c|}{ Hypertension } & $394(85.6)$ \\
\hline \multicolumn{2}{|c|}{ Diabetes mellitus } & $182(27.8)$ \\
\hline \multicolumn{2}{|c|}{ Hypercholesterolemia } & $376(39.6)$ \\
\hline \multicolumn{2}{|c|}{ Atrial fibrillation } & $91(19.8)$ \\
\hline \multicolumn{2}{|c|}{ Chronic obstructive pulmonary disease } & $68(14.8)$ \\
\hline \multicolumn{2}{|c|}{ Peptic ulcer disease } & $42(9.1)$ \\
\hline \multicolumn{2}{|c|}{ Thyroid disorders } & $67(14.6)$ \\
\hline \multicolumn{2}{|c|}{ Previous transient ischemic attack } & $5(1.1)$ \\
\hline \multicolumn{2}{|c|}{ Previous stroke } & $35(7.6)$ \\
\hline \multicolumn{2}{|c|}{ Family history of early cardiovascular disease } & $118(25.7)$ \\
\hline \multicolumn{2}{|c|}{ Presence of implantable pacemaker } & $25(5.4)$ \\
\hline \multicolumn{2}{|c|}{ Presence of cardiac resynchronization therapy device } & $7(1.5)$ \\
\hline \multicolumn{2}{|c|}{ Presence of implantable cardioverter defibrillator } & $11(2.4)$ \\
\hline
\end{tabular}

Data are presented as number (percentage) unless otherwise indicated.

Abbreviations: CCS, Canadian Cardiovascular Society grading of angina pectoris; IQR, interquartile range; NYHA, New York Heart Association Functional Classification

moderate, and high. Data regarding tobacco consumption (a history of smoking, daily tobacco intake, and current tobacco use status) were also collected. Finally, the type of pharmacotherapy used by the patient on the day of admission to the hospital was noted.

The study protocol conformed to the ethical guidelines of the 1975 Declaration of Helsinki Oral informed consent was provided by all participants.

Statistical analysis The data were presented in agreement with the EQUATOR (Enhancing the Quality and Transparency Of health
Research) guidelines and analyzed according to the established statistical protocols. Continuous variables were presented as mean (SD) or median with interquartile range (IQR) and categorical variables as numbers and percentages. The Shapiro-Wilk test was used to determine if the quantitative data were normally distributed. The $t$ test, Mann-Whitney test, and $X^{2}$ test were used for statistical comparisons. A $P$ value of less than 0.05 was considered significant. The STATISTICA software, version 13.1 (StatSoft Inc., Tulsa, Oklahoma, United States) was used to perform statistical analyses.

RESULTS Baseline characteristics of the study population A total of 460 patients (women, 26.1\%) at a mean (SD) age of 66.4 (9.9) years were enrolled in this study. Among them, $62.4 \%$ had at least 1 myocardial infarction and $26.1 \%$ had a history of 2 or more myocardial infarctions. Previous percutaneous coronary intervention and coronary artery bypass grafting were performed in $81.1 \%$ and $10.7 \%$ of patients, respectively. Baseline characteristics and comorbidities of the patients are shown in TABLE 1.

Body composition The median (IQR) recorded BMI was 28 (25.8-31.3) kg/m²; precisely, $0.4 \%$ of patients were underweight, $19.3 \%$ were within normal range of weight, $46.3 \%$ were overweight, and $33.9 \%$ were obese (class I, 25\%; class II, 7.6\%; class III, $1.3 \%$ ). This indicated that $80.7 \%$ of all patients $(82.9 \%$ of men and $74.2 \%$ of women; $P=0.04$ ) did not comply with the BMI recommendations for secondary prevention of coronary artery disease (TABLE2).

Based on waist circumference measurements, abdominal overweight was found in $26.7 \%$ of patients (30.3\% of men and $16.7 \%$ of women; $P=0.004)$ and abdominal obesity in $38.5 \%$ (32.6\% of men and $55 \%$ of women; $P<0.001$ ).

Blood glucose levels In the study population, $39.6 \%$ of patients were diabetic; $51.1 \%$ of them were treated only with oral diabetes medications, $20.3 \%$ with a combination of oral antidiabetic agents and insulin therapy, $25.3 \%$ only with insulin injections, and $3.3 \%$ only with diet. The median (IQR) fasting glucose level in nondiabetic patients was $5.4(5-5.8) \mathrm{mmol} / \mathrm{l}$. Among the nondiabetic patients, $43.5 \%$ ( $46.7 \%$ of men and $33.3 \%$ of women; $P=0.056$ ) had a fasting blood glucose level higher than $5.5 \mathrm{mmol} / \mathrm{l}$ and in $2.9 \%$ of cases (only men) this level was greater than or equal to $7 \mathrm{mmol} / \mathrm{l}$. In diabetic patients, the $\mathrm{HbA}_{1 \mathrm{c}}$ level was examined and the median (IQR) value was $7.2 \%(6.2 \%-8.1 \%)$, with $55.5 \%$ of diabetic patients $(50.8 \%$ of men and $66.7 \%$ of women; $P=0.049$ ) having the $\mathrm{HbA}_{1 c}$ level exceeding $7 \%$. Differences in the levels of blood glucose according to sex are shown in TABLE2. 
TABLE 2 Results of the obtained measurements and the rate of patients not adhering to the guidelines for secondary prevention of cardiovascular disease

\begin{tabular}{|c|c|c|c|c|}
\hline Variable & All patients $(n=460)$ & Men $(n=340)$ & Women $(n=120)$ & $P$ value \\
\hline Age, $y$, mean (SD) & $66.4(9.9)$ & $65.7(10)$ & $68.2(9.4)$ & 0.02 \\
\hline Body mass index, $\mathrm{kg} / \mathrm{m}^{2}$, median (IQR) & $28(25.8-31.3)$ & $28(25.9-31.1)$ & $28(24.9-31.7)$ & 0.99 \\
\hline Obese or overweight patientsa,$\%$ & 80.2 & 82.4 & 74.2 & 0.053 \\
\hline Waist circumference, cm, mean (SD) & $96.3(12.1)$ & $98.5(11.1)$ & $89.7(12.8)$ & $<0.001$ \\
\hline Abdominal overweight or obesity ${ }^{b}, \%$ & 65.2 & 62.9 & 71.7 & 0.08 \\
\hline $\begin{array}{l}\text { Fasting blood glucose level in nondiabetic patients, } \mathrm{mmol} / \mathrm{l} \text {, } \\
\text { median (IQR) }\end{array}$ & $5.4(5-5.8)$ & $5.4(5-5.9)$ & $5.3(5-5.6)$ & 0.08 \\
\hline $\begin{array}{l}\text { Fasting blood glucose level exceeding the recommendations } \\
(>5.5 \mathrm{mmol} / \mathrm{l}), \%\end{array}$ & 43.5 & 46.7 & 33.3 & 0.056 \\
\hline $\mathrm{HbA}_{1 \mathrm{c}}$ in diabetics, $\%$, median (IQR) & $7.2(6.2-8.1)$ & $7.1(6.2-8)$ & $7.4(6.3-8.5)$ & 0.16 \\
\hline $\mathrm{HbA}_{1 \mathrm{c}}$ in diabetics exceeding the recommendations $(>7 \%), \%$ & 55.5 & 50.8 & 66.7 & 0.049 \\
\hline Total cholesterol, mmol/l, median (IQR) & $3.8(3.1-4.6)$ & $3.7(3.1-4.4)$ & $4(3.5-5.2)$ & 0.001 \\
\hline Total cholesterol >5.2 mmol/l, \% & 13.5 & 9.7 & 24.2 & $<0.001$ \\
\hline LDL cholesterol, mmol/l, median (IQR) & $1.9(1.4-2.6)$ & $1.8(1.4-2.5)$ & $2(1.6-3.1)$ & 0.02 \\
\hline LDL cholesterol $\geq 1.4 \mathrm{mmol} / \mathrm{l}, \%$ & 78.7 & 77.4 & 82.5 & 0.24 \\
\hline HDL cholesterol, mmol/l, median (IQR) & $1.1(0.9-1.3)$ & $1.1(0.9-1.3)$ & $1.2(1-1.6)$ & 0.001 \\
\hline $\mathrm{HDL}$ cholesterol $\leq 1 \mathrm{mmol} / \mathrm{l}$ in men and $\leq 1.2 \mathrm{mmol} / \mathrm{l}$ in women, $\%$ & 43.9 & 41.2 & 51.7 & 0.047 \\
\hline Triglycerides, mmol/l, median (IQR) & $1.3(1-1.8)$ & $1.3(1-1.9)$ & $1.3(1-1.7)$ & 0.73 \\
\hline Triglycerides $>1.7 \mathrm{mmol} / \mathrm{l}, \%$ & 30.2 & 31.2 & 27.5 & 0.45 \\
\hline Systolic blood pressure, mm Hg, median (IQR) & $135(120-147)$ & $134(120-146.3)$ & $137.5(120-148.3)$ & 0.61 \\
\hline Systolic blood pressure $\geq 140 \mathrm{~mm} \mathrm{Hg}, \%$ & 40 & 37.6 & 46.7 & 0.08 \\
\hline Diastolic blood pressure, mm Hg, median (IQR) & $75(69-84)$ & $77(70-84)$ & $72(64.8-80)$ & 0.005 \\
\hline Diastolic blood pressure $\geq 90 \mathrm{~mm} \mathrm{Hg}, \%$ & 11.7 & 12.4 & 10 & 0.48 \\
\hline
\end{tabular}

a Body mass index $>25 \mathrm{~kg} / \mathrm{m}^{2}$

b Waist circumference $>95 \mathrm{~cm}$ in men and $>81 \mathrm{~cm}$ in women

Abbreviations: $\mathrm{HbA}_{1 \mathrm{c}}$ glycated hemoglobin; HDL, high-density lipoprotein; LDL, low-density lipoprotein; others, see TABLE 1

Blood lipid levels The median (IQR) level of total cholesterol was $3.8(3.1-4.6) \mathrm{mmol} / \mathrm{l}$ and it exceeded the desirable level in $13.5 \%$ of patients $(9.7 \%$ of men and $24.2 \%$ of women; $P<0.001)$. The median (IQR) level of LDL cholesterol was 1.9 (1.4-2.6) mmol/l, and it was higher than the level recommended by the guidelines in $78.7 \%$ of patients $(77.4 \%$ of men and $82.5 \%$ of women; $P=0.24$ ). The level of HDL cholesterol (median [IQR], 1.1 [0.9-1.3] mmol/l) was below the recommended level in $43.9 \%$ of patients ( $41.2 \%$ of men and $51.7 \%$ of women; $P=0.047$ ), and the level of triglycerides (median [IQR], 1.3 [1-1.8] mmol/l) was over the reference range in $30.2 \%$ of patients (31.2\% of men and $27.5 \%$ of women; $P=0.45$ ) (TABLE2).

Blood pressure The median (IQR) value of systolic blood pressure was 135 (120-147) mm Hg, while for diastolic pressure it was 75 (69-84) mm Hg. Systolic blood pressure greater than or equal to $140 \mathrm{~mm} \mathrm{Hg}$ was observed in $40 \%$ of patients $(37.6 \%$ of men and $46.7 \%$ of women; $P=0.08$ ) and diastolic blood pressure greater than or equal to $90 \mathrm{~mm} \mathrm{Hg}$ was noted in $11.7 \%$ (12.4\% of men and $10 \%$ of women; $P=$ $0.48)$. Systolic and / or diastolic blood pressure greater than or equal to $140 / 90 \mathrm{~mm} \mathrm{Hg}$ was recorded in $41.3 \%$ of patients $(39.1 \%$ of men and $47.5 \%$ of women; $P=0.11$ ) (TABle 2 ).

Dietary habits, physical activity, and tobacco use Low level of physical activity was noted in $56.7 \%$ of the studied patients $(61.5 \%$ of men and $43.3 \%$ of women), moderate in $36.7 \%$ ( $31.2 \%$ of men and $52.5 \%$ of women), and high in $6.5 \%$ ( $7.4 \%$ of men and $4.2 \%$ of women). A difference in the level of physical activity was found between the sexes $(P<0.001)$.

Dietary habits were assessed using study-dedicated questionnaires. Among all studied patients, $76.5 \%$ ( $75.3 \%$ of men and $80 \%$ of women) had changed their diet after the diagnosis 


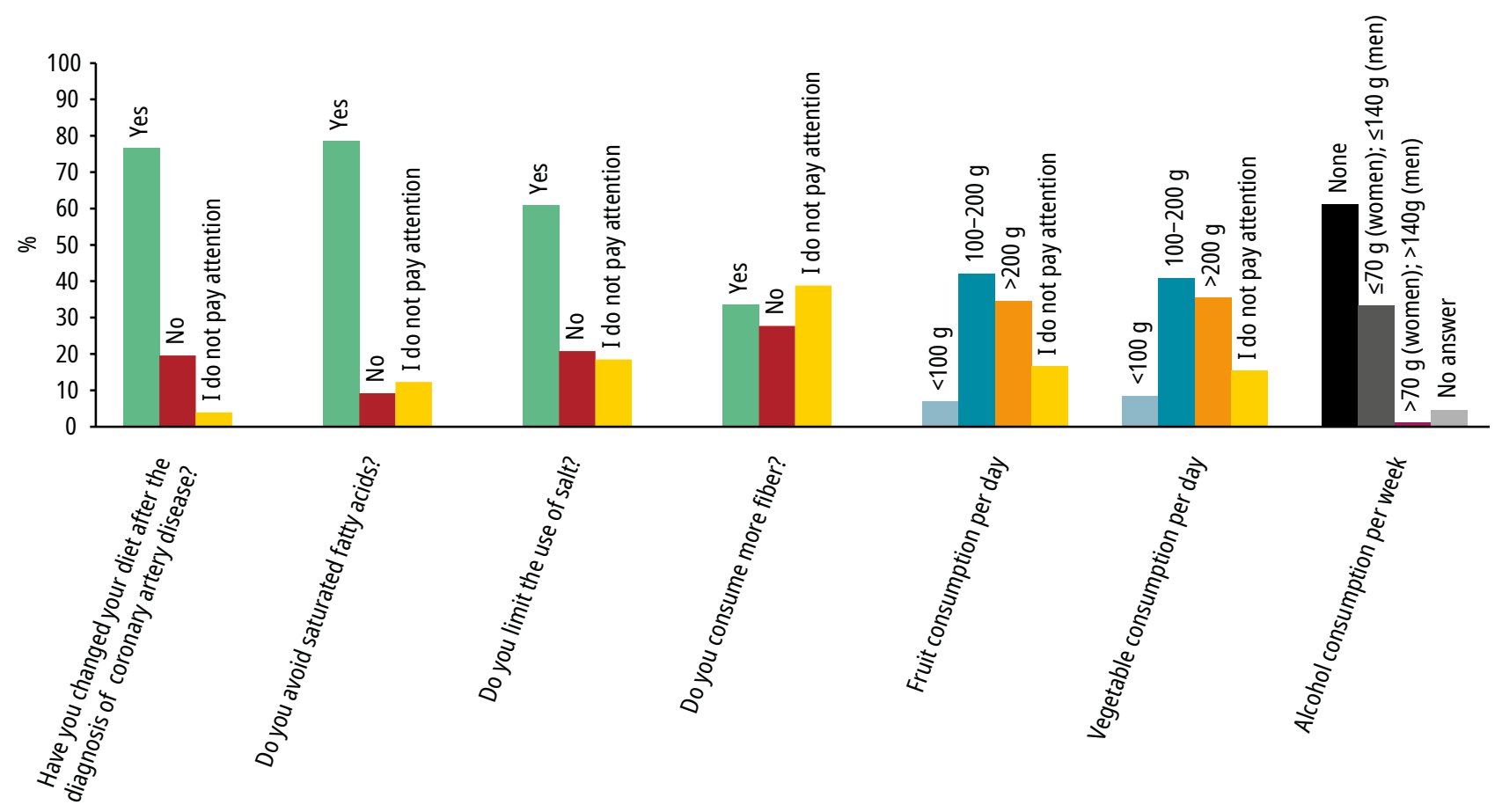

FIGURE 1 Results of the questionnaire regarding dietary habits

of coronary artery disease. Interestingly, 3.9\% of patients did not care about the diet $(4.7 \%$ of men and $1.7 \%$ of women). There was no difference between the sexes with respect to dietary changes $(P=0.28)$. Additionally, $78.5 \%$ of patients $(77.4 \%$ of men and $81.7 \%$ of women; $P=0.29)$ declared that they avoided the consumption of saturated fatty acids. A total of $60.9 \%$ of patients $(58.5 \%$ of men and $67.5 \%$ of women; $P=0.08$ ) reduced the consumption of salt. Moreover, $33.5 \%$ of patients $(32.6 \%$ of men and $35.8 \%$ of women; $P=0.52$ ) declared an increase in fiber consumption. Only $34.6 \%$ of patients $(34.4 \%$ of men and $35 \%$ of women; $P=0.92$ ) consumed over $200 \mathrm{~g}$ of fruit per day and $35.4 \%$ consumed over $200 \mathrm{~g}$ of vegetables per day $(30.6 \%$ of men and $49.2 \%$ of women, $P<0.001)$. Fish consumption was low among the study population: only $21.1 \%$ of participants consumed fish at least 2 times per week $(20 \%$ of men and $24.2 \%$ of women; $P=0.33$ ) and $18.7 \%$ of patients $(17.9 \%$ of men and $20.8 \%$ of women, $P=0.57$ ) did not eat fish at all. More than a half of the patients $(61.1 \% ; 51.2 \%$ of men and $89.2 \%$ of women; $P<0.001)$ declared that they did not drink alcohol (FIGURE 1 ).

Although $14.6 \%$ of patients ( $15.3 \%$ of men and $12.5 \%$ of women; $P=0.46$ ) admitted that they were current smokers, $41 \%$ of all studied individuals used to be tobacco users, with a history of median (IQR) 29 (15-40) pack-years. Fortunately, $63.2 \%$ of former smokers decided to give up smoking after developing symptoms of cardiovascular disease, and the rest cut down tobacco usage.
Pharmacotherapy Aldosterone antagonists were used by $19 \%$ of patients $(19.7 \%$ of men and $16.7 \%$ of women; $P=0.47$ ), $\beta$-blockers by $89.8 \%$ ( $89.4 \%$ of men and $90.8 \%$ of women; $P=0.66$ ), calcium channel blockers by $32 \%$ ( $28.9 \%$ of men and $39.2 \%$ of women; $P=0.04)$, angiotensin converting enzyme inhibitors by $68.7 \%$ ( $72.6 \%$ of men and $57.5 \%$ of women; $P=0.002)$, and angiotensin II receptor blockers by $13 \%$ ( $11.8 \%$ of men and $16.7 \%$ of women; $P=0.17)$. Diuretics were used by $48.9 \%$ ( $47.1 \%$ of men and $54.2 \%$ of women; $P=0.18)$ and statins by $93.5 \%$ of patients $(95 \%$ of men and $89.2 \%$ of women; $P=0.03$ ). Acetylsalicylic acid was taken by $89.6 \%$ of patients $(90 \%$ of men and $88.3 \%$ of women, $P=0.6$ ), while $50.2 \%$ of the group (54.4\% of men and $38.3 \%$ of women, $P=0.003$ ) were on dual antiplatelet therapy $(33.5 \%$ on clopidogrel and $18.5 \%$ on ticagrelor). A total of $10.4 \%$ of patients did not use any antiplatelet medications ( $10 \%$ of men and $11.7 \%$ of women; $P=0.6$ ) and $6.7 \%$ of individuals were on anticoagulant therapy due to atrial fibrillation $(6.8 \%$ of men and $6.7 \%$ of women; $P=0.97)$.

A combination of $\beta$ blocker or calcium channel blocker, angiotensin converting enzyme inhibitor or angiotensin II receptor blocker, antiplatelet or antithrombotic medications in case of atrial fibrillation, and a statin was used in $72.2 \%$ of patients $(75.3 \%$ of men and $63.3 \%$ of women; $P$ $=0.01$ ). Three medications from these groups were taken in $22.4 \%$ (20.3\% of men and $28.3 \%$ of women; $P=0.07$ ), 2 in $3.7 \%$ (2.9\% of men and $5.8 \%$ of women; $P=0.15$ ), and 1 in $1.3 \%$ of cases $(0.9 \%$ of men and $2.5 \%$ of women; $P=0.19$ ). In $0.4 \%$ of patients (only men), no pharmacotherapy was used. 


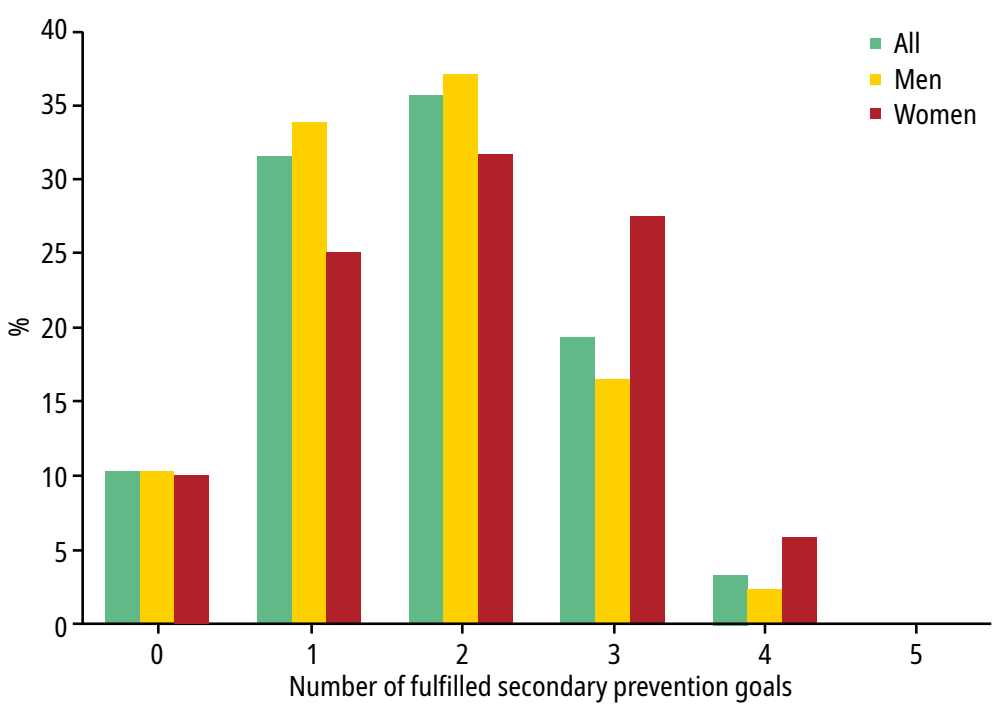

FIGURE 2 Number of fulfilled secondary prevention goals

Implementation level of guidelines The analysis comprised 5 secondary prevention goals which could be evaluated in the most objective way, that is: (1) normal weight (BMI 18.5-24.9 kg/m²); (2) no smoking status; (3) LDL cholesterol level below $1.4 \mathrm{mmol} / \mathrm{l}$; (4) normal levels of fasting glucose for patients without diabetes mellitus (3.9$5.5 \mathrm{mmol} / \mathrm{l}$ ) and $\mathrm{HbA}_{1 \mathrm{c}}$ for diabetic patients not exceeding 7\%; and (5) systolic blood pressure below $140 \mathrm{~mm} \mathrm{Hg}$ and diastolic blood pressure below $90 \mathrm{~mm} \mathrm{Hg}$. No patient fulfilled all the prevention goals. Overall, 4 of these goals were fulfilled by $3.3 \%, 3$ by $19.3 \%, 2$ by $35.7 \%$, and a single goal by $31.5 \%$ of patients. In $10.2 \%$ of cases none of the above-mentioned criteria were achieved. A difference in the implementation level of guidelines was found between the sexes, with men being worse responders than women $(P<0.001)$ (FIGURE2).

DISCUSSION Our study clearly showed that the level of adherence to the guidelines for secondary prevention of coronary artery disease was extremely low. Most of the patients (80.7\%) did not comply with the body weight recommendations, being either overweight or obese, which, in combination with a low level of physical activity (declared by $56.7 \%$ of the studied patients) and bad dietary habits (low consumption of fiber, fish, fruit, and vegetables), significantly increased cardiovascular risk. Moreover, almost half of nondiabetic patients $(43.5 \%)$ had fasting blood glucose level exceeding $5.5 \mathrm{mmol} / \mathrm{l}$ and more than a half of diabetic patients $(55.5 \%)$ had the $\mathrm{HbA}_{1 c}$ level higher than 7\%. Also, hyperlipidemia was a considerable problem in the studied cohort, especially the level of LDL cholesterol, which was exceeded in over three-fourths of patients ( $78.7 \%$ of cases). Insufficient management of arterial blood pressure was also detected in almost half of patients (41.3\%). Fortunately, considerable positive changes in eating habits and tobacco use could be observed; $76.5 \%$ of patients declared that they had changed their diet after the diagnosis of coronary artery disease and as much as $63.2 \%$ of former smokers decided to give up smoking after developing symptoms of cardiovascular disease.

Much evidence has shown that a large proportion of cardiovascular disease burden could be explained by behavioral factors, meanwhile our results and other large studies showed that a large majority of coronary disease patients had unhealthy lifestyles., ${ }^{719}$ Promoting a healthy lifestyle (eg, physical activity, healthy diet, avoiding alcohol and tobacco use) has been crucial for the primary and secondary prevention of coronary artery disease. A dose-response relationship between physical activity and cardiovascular disease morbidity and mortality has been described. ${ }^{20}$ However, there has not been a consensus on an appropriate method of defining the level of physical activity during medical control. Nevertheless, patients should be encouraged to be as physically active as possible. Although a majority of patients declared a change in their diet since hospitalization, more specific questions showed poor adherence to the recommended healthy diet characteristics. This highlighted the need to introduce targeted dietary advice promoting heart-healthy eating habits that should be obligatory for all patients. One of the significant lifestyle factors was the consumption of tobacco. It was comforting that only $14.6 \%$ of patients from our cohort were active smokers; on the other hand, $41 \%$ of individuals smoked before the occurrence of cardiovascular events. Quitting smoking was associated with a substantial reduction in the risk of all-cause mortality among patients with diagnosed coronary heart disease and could be achieved relatively easy. ${ }^{21} \mathrm{~A}$ recent study by Siudak et al ${ }^{22}$ showed that constant education of patients after myocardial infarction was an effective method of smoking cessation in over $50 \%$ of smokers 6 months after myocardial infarction.

Hypertension has been one of the most significant risk factors for the development of coronary artery disease. A proper management of hypertension might significantly reduce the risk for secondary events. The SPRINT study (Systolic Blood Pressure Intervention Trial) revealed that in nondiabetic patients at a high risk of cardiovascular events, a target systolic blood pressure of less than $120 \mathrm{~mm} \mathrm{Hg}$, as compared with less than $140 \mathrm{~mm} \mathrm{Hg}$, resulted in $25 \%$ reduction of major cardiovascular events and $27 \%$ reduction of death from any cause. No significant decrease in stroke or myocardial infarction rate was detected. ${ }^{23}$ Unattended automated office blood pressure measurement was used in this study, which gives lower values than traditional methods of measurement. Thus, this value might correspond to conventional office systolic blood pressure in 
the range of 130 to 140 versus 140 to $150 \mathrm{~mm} \mathrm{Hg} .^{16}$ Moreover, a large meta-analysis of 147 randomized trials showed that a reduction of systolic blood pressure by $10 \mathrm{~mm} \mathrm{Hg}$, or diastolic by $5 \mathrm{~mm} \mathrm{Hg}$, reduced coronary heart disease events by about one-quarter and stroke by about one-third. ${ }^{24} \mathrm{Un}$ fortunately, the recommended value of blood pressure was not achieved by $41.3 \%$ of our patients. Moreover, if we had taken a more restricted target for patients with diabetes $(<130 / 80 \mathrm{~mm} \mathrm{Hg})$, as much as $69.8 \%$ of individuals from our studied cohort would not have reached the recommended levels. That indicated that more actions should be taken by both patients and clinicians to manage this important risk factor.

The current study found significant sex-related differences in the implementation of the guidelines. Generally, men were worse responders than women. Nevertheless, among all studied factors, diabetic women achieved worse glycemic control than men and they had worse control of total cholesterol and HDL fraction cholesterol levels.

Strenuous efforts should be made to improve the adherence to secondary prevention guidelines, which would result in slowing the progression of the disease, reducing secondary events and mortality, improving the quality of life, and reducing the number of hospitalizations. ${ }^{7,19}$ Moreover, optimized and streamlined patient care may reduce the use of personnel and financial resources. ${ }^{25}$ Most cardiovascular risk factors could be modified and controlled. In our opinion, frequent control visits to a specialist or general practitioner would enable to improve the obtained results. Currently, this measure is already implemented in Poland for patients after a myocardial infarction. Continued follow-up visits and repeated blood tests will allow to reduce the risk of the recurrence of cardiovascular events. A combination of patients' education and professional and practical advice on the possibilities of lifestyle change and regular medical control should be implemented during any contact with medical staff. Moreover, nationwide secondary prevention programs and advertising campaigns directed to patients should be intensified to improve the processes of care. To increase the level of implementation, the process should be monitored by audits, surveys, or by assessing outcome data. ${ }^{25}$ On the other hand, physicians must be regularly trained and educated on how to properly implement the guidelines into their clinical practice. ${ }^{26}$ Finally, social media and mobile medical software applications may be used as powerful and widely available tools to help implement the clinical guidelines. ${ }^{27}$

Limitations There are 2 main limitations of the study that should be considered. First, this was a single-center study focused on a local population of patients, therefore the level of adherence to the guidelines for secondary prevention of coronary artery disease might be different in other centers. Second, not all but only the main, arbitrarily chosen factors that had an influence on the development of cardiovascular disease, were analyzed. Nevertheless, we believe that these limitations did not significantly impede our analyses and that the derived conclusions may be extrapolated to the general population.

Conclusions The level of adherence to the guidelines for secondary prevention of coronary artery disease was extremely low. No patient fulfilled all the prevention goals and in $10.2 \%$ of cases none of the analyzed recommendations were achieved. Significant difference in the implementation level of the guidelines was found between the sexes, with men being worse responders than women. More attention should be paid to proper implementation of the guidelines and patients' education to prevent further development of cardiovascular disease.

\section{SUPPLEMENTARY MATERIAL}

Supplementary material is available at www.mp.pl/kardiologiapolska.

\section{ARTICLE INFORMATION}

\section{CONFLICT OF INTEREST None declared.}

OPEN ACCESS This is an Open Access article distributed under the terms of the Creative Commons Attribution-NonCommercial-NoDerivatives 4.0 International License (CC BY-NC-ND 4.0), allowing third parties to download articles and share them with others, provided the original work is properly cited, not changed in any way, distributed under the same license, and used for noncommercial purposes only. For commercial use, please contact the journal office at kardiologiapolska@ptkardio.pl.

HOW TO CITE Krawczyk-Ożóg A, Płotek A, Hołda M, et al. Assessment of the implementation level of the guidelines for secondary prevention of cardiovascular disease in everyday clinical practice. Kardiol Pol. 2021; 79: 434-441. doi:10.33963/KP.15856

\section{REFERENCES}

1 Piepoli MF, Hoes AW, Agewall S, et al. New European guidelines for cardiovascular disease prevention in clinical practice. Atherosclerosis. 2016; 252: 207-274.

2 Moran AE, Forouzanfar MH, Roth GA, et al. Temporal trends in ischemic heart disease mortality in 21 world regions, 1980 to 2010. Circulation. 2014; 129: 1483-1492.

3 Kaasenbrood L, Boekholdt SM, van der Graaf Y, et al. Distribution of estimated 10-year risk of recurrent vascular events and residual risk in a secondary prevention population. Circulation. 2016; 134: 1419-1429.

4 Knuuti J, Wijns W, Saraste A, et al. 2019 ESC guidelines for the diagnosis and management of chronic coronary syndromes. Eur Heart J. 2020; 41: 407-477.

5 Wójcicki K, Krycińska R, Tokarek T, et al. Knowledge and prevalence of risk factors for coronary artery disease in patients after the first and repeated percutaneous coronary intervention. Kardiol Pol. 2020; 78:147-153.

6 Kotseva K, Wood D, de Backer G, et al. EUROASPIRE III. Management of cardiovascular risk factors in asymptomatic high-risk patients in general practice: cross-sectional survey in 12 European countries. Eur J Prev Cardiol. 2010; 17: 530-540.

7 Kotseva K, Wood D, De Bacquer D, et al. EUROASPIRE IV: a European Society of Cardiology survey on the lifestyle, risk factor and therapeutic management of coronary patients from 24 European countries. Eur J Prev Cardiol. 2016; 23: 636-648.

8 Kotseva K, Wood D, De Backer G, et al. Cardiovascular prevention guidelines in daily practice: a comparison of EUROASPIRE I, II, and III surveys in eight European countries. Lancet. 2009; 373: 929-940.

9 Vilaubí JMP, Orozco-Beltrán D, Gonçalves AQ, et al. Adherence to European clinical practice guidelines for secondary prevention of cardiovascular disease: a cohort study. Int J Environ Res Public Health. 2018; 15: 1233.

10 Cordero A, Rodriguez Padial L, Batalla A, et al. Optimal pharmacological treatment and adherence to medication in secondary prevention of cardiovascular events in Spain: results from the CAPS study. Cardiovasc Ther. 2017; 35: e12240. 
11 Executive summary of the clinical guidelines on the identification, evaluation, and treatment of overweight and obesity in adults. Arch Intern Med. 1998; 158: $1855-1867$.

12 World Health Organization. Waist circumference and waist-hip ratio: report of a WHO expert consultation, Geneva, December 8-11, 2008. https:// apps.who.int/iris/bitstream/handle/10665/44583/9789241501491_eng. pdf?sequence=1\&isAllowed=y. Accessed June 1, 2016.

13 Mach F, Baigent C, Catapano AL, et al. 2019 ESC/EAS Guidelines for the management of dyslipidaemias: lipid modification to reduce cardiovascular risk. Eur Heart J. 2020; 41: 111-188

14 Piepoli MF, Hoes AW, Agewall S, et al. 2016 European Guidelines on cardiovascular disease prevention in clinical practice. Eur Heart J. 2016; 37: 2315-2381.

15 Araszkiewicz A, Bandurska-Stankiewicz E, Budzyński A, et al. 2019 Guidelines on the management of diabetic patients. A position of Diabetes Poland. Clinical Diabetology. 2019; 8: 1-95.

16 Williams B, Mancia G, Spiering W, et al. 2018 ESC/ESH Guidelines for the management of arterial hypertension. Eur Heart J. 2018; 39: 3021-3104.

17 International Physical Activity Questionnaire [in Polish]. https://sites. google.com/site/theipaq/questionnaire_links/IPAQ_Polish_self-admin_short. pdf?attredirects $=0 \& d=1$. Accessed June 1, 2016.

18 International Physical Activity Questionnaire (October 2002). Long last 7 days self-administered format for use with young and middle-aged adults (15-69 years). https://www.physio-pedia.com/images/6/6e/International_Physical_Activity_Questionaire.pdf. Accessed June 1, 2016.

19 Kotseva K, De Backer G, De Bacquer D, et al. Lifestyle and impact on cardiovascular risk factor control in coronary patients across 27 countries: results from the European Society of Cardiology ESC-EORP EUROASPIRE V registry. Eur J Prev Cardiol. 2019; 26: 824-835.

20 Carnethon MR. Physical activity and cardiovascular disease: how much is enough? Am J Lifestyle Med. 2009; 3: 44S-49S.

21 Critchley JA, Capewell S. Mortality risk reduction associated with smoking cessation in patients with coronary heart disease: a systematic review. JAMA. 2003; 290: 86-97.

22 Siudak Z, Krawczyk-Ozióg A, Twarda I, et al. "Heart without smoke" educational campaign - the role of patient education in secondary prevention of cardiovascular disease. Kardiol Pol. 2018; 76: 125-129.

23 SPRINT Research Group; Wright JT Jr, Williamson JD, Whelton PK, et.al. A randomized trial of intensive versus standard blood-pressure control. N Engl J Med. 2015; 373: 2103-2116.

24 Law MR, Morris JK, Wald NJ. Use of blood pressure lowering drugs in the prevention of cardiovascular disease: meta-analysis of 147 randomised trials in the context of expectations from prospective epidemiological studies. BM]. 2009; 338: b1665.

25 Baron DM, Metnitz PGH, Rhodes A, et al. Clinical guidelines. How can we improve adherence and implementation? Eur J Anaesthesiol. 2017; 34: 329-331.

26 Shekelle $\mathrm{P}$, Woolf S, Grimshaw JM, et al. Developing clinical practice guidelines: reviewing, reporting, and publishing guidelines; updating guidelines; and the emerging issues of enhancing guideline implementability and accounting for comorbid conditions in guideline development. Implement Sci. 2012; 7: 62 .

27 Ventola CL. Mobile devices and apps for health care professionals: uses and benefits. P T. 2014; 39: 356-364. 